\title{
Glycogen is the primary source of glucose during the lag phase of E. coli proliferation
}

Tomoaki Yamamotoya ${ }^{\mathrm{a}, 1,2}$, Hitomi Dose ${ }^{\mathrm{b}, 1}$, Zhongyuan Tian ${ }^{\mathrm{a}}$, Adrien Fauréa ${ }^{\mathrm{a}}$, Yoshihiro Toya ${ }^{\mathrm{c}}$, Masayuki Honma ${ }^{\mathrm{c}}$, Kaori Igarashi ${ }^{\mathrm{c}}$, Kenji Nakahigashic ${ }^{\mathrm{c}}$, Tomoyoshi Soga ${ }^{\mathrm{c}}$, Hirotada Mori ${ }^{\mathrm{b},}$, Hiroshi Matsuno ${ }^{\mathrm{a},{ }^{*}}$

${ }^{a}$ Graduate School of Science and Engineering, Yamaguchi University, 1677-1 Yoshida, Yamaguchi-shi, Yamaguchi 753-8512, Japan

${ }^{b}$ Graduate School of Biological Sciences, Nara Institute of Science and Technology, 8916-5 Takayama, Ikoma, Nara 630-0101, Japan

${ }^{c}$ Institute for Advanced Biosciences, Keio University, Tsuruoka, Yamagata 997-0017, Japan

\begin{abstract}
In the studies of E. coli (Escherichia coli), metabolomics analyses have mainly been performed using steady state culture. However, to analyze the dynamic changes in cellular metabolism, we performed a profiling of concentration of metabolites by using batch culture. As a first step, we focused on glucose uptake and the behavior of the first metabolite, G6P (glucose-6-phosphate). A computational formula was derived to express the glucose uptake rate by a single cell from two kinds of experimental data, extracellular glucose concentration and cell growth, being simulated by Cell Illustrator. In addition, average concentration of G6P has been measured by CE-MS. The existence of another carbon source was suggested from the computational result. After careful comparison between cell growth, G6P concentration, and the computationally obtained curve of glucose uptake rate, we predicted the consumption of glycogen in lag phase
\end{abstract}

\footnotetext{
Abbreviations: 3PG, 3-phosphoglycerate; 6PG, 6-phosphogluconate; CSML, cell system markup language; F6P, fructose-6-phosphate; G1P, glucose-1-phosphate; G6P, glucose-6-phosphate; HFPN, hybrid functional Petri net; OD, optical density; PEP, phosphoenolpyruvate; PPP, pentosephosphate pathway; PTS, phosphotransferase system; TCA, tricarboxylic acid.

1 These authors contributed equally and are considered as co-first authors.

2 Current Address: Hitachi Solutions, Ltd., 4-12-7 Higashishinagawa, Shinagawa-ku, Tokyo 140-0002, Japan.

* Corresponding Authors: H. Mori: hmori@gtc.naist.jp, Tel. +81-743-72-5660, Fax +81-743-72-5669; H. Matsuno: matsuno@sci.yamaguchi-u.ac.jp, Tel. \& Fax+81-83-933-5697.
} 
and its accumulation as an energy source in an E. coli cell for the next proliferation. We confirmed our prediction experimentally. This behavior indicates the importance of glycogen participation in the lag phase for the growth of E. coli.

\section{Keywords:}

cell growth; computational simulation; E. coli metabolism; glycogen

\section{Introduction}

Integrative process of biological experiments and computational modeling produces a synergistic effect on the systematic understanding of cellular systems. For the study of metabolic systems, E. coli (Escherichia coli) has been extensively used [1]. Response to a pulse of glucose was examined by a dynamic model of the central carbon metabolism [2]. A kinetic model of enzyme network control enabled the quantitative analysis of ammonium assimilation regulation $[3,4]$. Using a computational model that couples enzymatic and transcriptional regulation of the central metabolism, system-wide adjustment of metabolic operation between glycolytic and gluconeogenic carbon sources were explained [5]. An ordinary differential equation based mathematical model was built to illustrate the relation between the growth rate and the output of the sensor system PEP (phosphoenolpyruvate) dependent PTS (phosphotransferase system) [6,7].

In the studies of E. coli, including aforementioned studies, metabolomics analyses have mainly been conducted by continuous culture. However, this method does not allow us to observe the transition of bacterial growth phases, i.e., the sequence of the starting point, log phase, and stationary phase. Aiming at the systematic understanding of $E$. coli growth mechanism, we have started a study to establish a computational model, based on the measured expression patterns of metabolites in batch culture.

As a first step, we focus on average cellular glucose uptake. For this, we have measured three basic parameters at the entry point of glucose into a cell: (I) glucose consumption, (II) bacterial growth, and (III) average cellular G6P concentration. 
Glucose is a simple sugar and an important carbohydrate in most of organisms. Cells use this sugar as the primary source of energy and as a precursor for the synthesis of several important metabolites. The first step of glucose utilization is the transportation from the environment with phosphorylation to convert glucose to G6P by PEP (phosphoenolpyruvate) dependent PTS, reviewed by Gosset [8]. Then, G6P is metabolized by glycolysis through F6P (fructose-6-phosphate), PPP (pentosephosphate pathway) through 6PG (6-phosphogluconate) or glycogen production through G1P (glucose-1-phosphate). Glycolysis and PPP provide essential metabolism for production of energy and intermediates for essential biomolecules, such as amino acids, lipids, nucleotides etc.

The three parameters, (I), (II), and (III), have been used to describe the average cellular glucose uptake rate. The first two, glucose consumption and bacterial growth are used to derive a formula expressing the average cellular glucose uptake rate. With a computational model based on this formula, a behavior of G6P concentration is realized which has been tested by experimental measurement. Comparison between the computational and experimental results implies the existence of another sugar donor.

Glycogen is a well-known molecule that functions as energy storage in many organisms, being utilized when a sudden need for glucose emerges [9]. Numerous studies focused on glycogen storage diseases in human [10-16]. There are also many studies on glycogen network in prokaryotes [17-25], which serve as simple models of glycogen storage diseases in mammals, but most of them focus on glycogen network only. On the other hand, in the area of metabolic engineering, the most studied carbohydrate metabolic pathways are EMD (the Embden-Meyerhof-Parnas pathway), PPP and ED (the Entner-Doudoroff pathway) [26], but seldom glycogen network was taken into account in biological or computational central metabolism models [27-35]. Though Traxler et al. showed much valuable high throughput data of both glycogen network and other carbohydrate metabolic networks [36-38], their main focus was on ppGpp. Preiss et al. and Sigal et al. have carefully studied the relationship of glucose, glycogen and cell growth $[39,40]$, however they did not extend their research to downstream metabolites, G6P, G1P etc.

Currently, we are studying glycogen metabolic network together with glycolysis, 
and based on our results we made a set of new predictions for the role of glycogen in $E$. coli: (1) glycogen supplies sugar for cell activity in lag phase and (2) glucose is converted to glycogen as energy supply for immediate usage in the next growth phase of E. coli. The conjectured behavior of glycogen concentration based on this prediction has been confirmed experimentally (section 2.3). Furthermore, the computational model for G6P concentration was updated so that this confirmed glycogen participation could be realized, thus solving the discrepancy between computational and experimental results.

These results show that, in E. coli, both cellular glycogen and extracellular glucose act as primary sugar donors, which alternatively function at lag phase and log phase. Accordingly we took glycogen metabolic pathway into account in our computational model of E. coli central metabolism pathway, in which glycogen plays an important, though non essential role $[17,20,23]$.

\section{Materials and methods}

\subsection{Bacterial strain, media and culture condition}

E. coli $\mathrm{K}-12 \mathrm{~W} 3110\left(\mathrm{~F}-\mathrm{IN}(\mathrm{rrnD}-\mathrm{rrnE}) 1 \lambda^{-}\right)$was mainly used in this study. MOPS medium [41] supplemented with $0.4 \%$ glucose was used for bacterial culture. $1 \mathrm{ml}$ of overnight culture was inoculated into $100 \mathrm{ml}$ fresh media in $500 \mathrm{ml}$ flask and cultured at 37 with shaking $(180 \mathrm{rpm})$. Cell growth was monitored by optical density at $600 \mathrm{~nm}$ (OD600) by spectrophotometer DU-600 (Beckman Coulter, Inc., CA, USA).

\subsection{Measurement of glucose and ammonium concentration in culture media}

Glucose and ammonium concentration in medium were measured by Glucose CII kit (WAKO Co. Ltd., Osaka, Japan) and F-kit ammonia (Roche, Basel, Switzerland), respectively. Samples were collected every hour until 12 hours or every 3 hours up to 24 hours after starting culture. Cells were collected by centrifugation at $15,000 \mathrm{rpm}$ for 3 minutes. The supernatants were kept in freezer at -30 until measurement.

\subsection{Measurement of cellular glycogen concentration}

Cells were harvested by centrifugation, washed with MOPS medium without glucose and resuspended in ice-cold $1 \mathrm{ml} 40 \mathrm{mM}$ Tris- $\mathrm{HCl}(\mathrm{pH} 7.5)$. The cell suspension was 
sonicated 3 cycles of 45 seconds on ice. Glycogen was precipitated with $40 \%$ final concentration of ethanol, washed with $40 \%$ ethanol and resuspended in $250 \mathrm{ml}$ distilled water. After autoclaving at 130 for 1 hour, the intracellular glycogen concentration in the supernatant was measured by EnzyChrom Glycogen Assay Kit (BioAssay Systems, CA, USA) according to the manufacturer's instructions.

\subsection{Measurement of cellular metabolites concentration}

G6P, PEP and 3PG (3-phosphoglycerate) concentration were quantified by CE-MS (capillary electrophoresis mass spectrometry) analysis described by Soga et al. [42]. Samples were collected at 5, 6, 7,9 and 11 hours after starting of culture.

\subsection{Gompertz function}

The Gompertz function is a sigmoid function to simulate an S-shape curve; it is a type of mathematical model for a time series, where growth is slowest at the start and end of a time period $[43,44]$.

$$
y(t)=a e^{b e^{c t}}
$$

where $a$ is the upper asymptote, $b$ sets the horizontal-axis displacement, and $c$ sets the growth rate; $e$ is Euler's Number. A reverse Gompertz function was used to imitate inversed S-shape curve of extracellular glucose concentration, and of glycogen (sugar donor part) concentration; in addition, a forward Gompertz function was used for calculating cell proliferation curve.

\subsection{Cell Illustrator}

Cell Illustrator (Cell Illustrator 4.0: http://www.cellillustrator.com/ci40), a biological simulation software developed by the University of Tokyo, helps researchers to simulate complex biological processes, especially for biologists to visually analyze biological pathways. The solid reputation of Cell Illustrator comes from its very flexible performance on HFPN (hybrid functional Petri net), which is a strong tool to analyze and simulate biological networks dynamically, especially in the area of metabolism [45]. In this paper, our computational plots (Fig. 1(c), Fig. 4 etc.) were achieved from this 
software. Moreover, all the CSML (cell system markup language) models of this paper are available

in

our

website

(http://ds0n.cc.yamaguchi-u.ac.jp/ mzemi/etchp/TZ/AdditionalFiles.html).

\section{Results}

\subsection{Simulation of intracellular G6P concentration}

We have measured the time course behaviors of metabolites in E. coli cells by means of batch culture. According to KEGG (http://www.kegg.jp/) and MetaCyc (http://metacyc.org/), because the main sugar source of the glycolytic pathway is glucose, as a first step, we focused on G6P, to which glucose in a medium is directly converted. In order to simulate the evolution of intracellular G6P concentration during growth, we need to evaluate the average cellular glucose uptake speed, as a function of time. We first measured extracellular glucose concentration and OD (optical density) of a batch culture [46]. The results are shown in Fig. 1(a) and 1(b). We have separately showed that the OD curve behaves similarly as the number of cells. Bacterial proliferation curve mainly includes three phases: lag phase, log phase and stable phase; and glucose curve starts its exponential decreasing phase after a preparation phase.

These curves were approximated using Gompertz functions, whose parameters were fitted manually:

$$
\begin{aligned}
& M_{g l c}(t)=A\left(1-a_{1} e^{b_{1} e^{c_{1} t}}\right) \\
& C_{\text {cell }}(t)=B+a_{2} e^{b_{2} e^{c_{2} t}}
\end{aligned}
$$

Eqn (2) gives extracellular glucose concentration as a function of time; $A$ is the initial value of extracellular glucose concentration, and $a_{1}, b_{1}, c_{1}$ are parameters of the Gompertz function. Eqn (3) gives the kinetics function of cell proliferation of E. coli; $B$ denotes the initial value of cells number, and $a_{2}, b_{2}, c_{2}$ are cell proliferation associated parameters of the Gompertz function. Then global glucose uptake speed is given by: 


$$
V_{g l c}(t)=-M_{g l c}^{\prime}(t)
$$

where $M_{g l c}^{\prime}(t)=A a_{1} b_{1} c_{1} e^{\left(b_{1} e^{c^{l t}}+c_{1} t\right)}$ is the derivative of $M_{g l c}(t)$. Then dividing $V_{g l c}(t)$ by cell numbers $C_{\text {cell }}(t)$ gives average cellular glucose uptake speed over time, $v_{g l c}(t)$ :

$$
v_{g l c}(t)=k_{1} V_{g l c}(t) / C_{c e l l}(t)
$$

where $k_{1}$ is a manually fitting parameter to normalize the different units between results of different methods. We applied mass balance theory to design this simplified model of carbohydrate metabolism, with the aim of simulating G6P concentration. From this point of view, G6P accumulation speed equals to the difference between input speed and output speed of sugar group flowing through G6P:

$$
d\left(m_{G 6 P}\right) / d t=v_{g l c}^{\text {input }}-v^{\text {output }},
$$

where $v_{\text {glc }}^{\text {input }}$ represents the input speed of sugar group, which mainly comes from extracellular glucose; $v^{\text {output }}$ is the sugar group output speed, which reflects a combined speed of downstream fluxes from G6P, which include all major carbohydrate metabolic pathways associated with cell proliferation, such as glycolysis, PPP etc. Output transition speed $v^{\text {output }}=k_{2} m_{G 6 P}$ is determined by the law of mass action, with $k_{2}$, an ad hoc coefficient.

Transplanting aforementioned kinetics expressions in Eqn (6), by adjusting parameters to suitable values $\left(A=410, a_{1}=1, b_{1}=-45, c_{1}=-0.49, B=3, a_{2}=40\right.$, $\left.b_{2}=-30, c_{2}=-0.6, k_{1}=100, k_{2}=1.5\right)$, we got G6P concentration curve (Fig. 1(c)) by calculating these equations in Cell Illustrator (see section 2.6). This computational result predicts a single peak between 7 and $8 \mathrm{hr}$. We measured average G6P concentration by means of CE-MS at 5, 6, 7, 9, and $11 \mathrm{hr}$. The data is presented in Fig. 1(d), which shows a trough (called the 'hook' here) followed by a peak at about $7 \mathrm{hr}$ (the 'second peak' in this paper). Thus we consider that it is reasonable if the computational peak appears within 6 and $9 \mathrm{hr}$. Hence the correctness of the location of the computed peak was confirmed by the second peak of the G6P experimental data. 


\subsection{Extracellular glucose is not the only sugar donor}

The behavior of G6P concentration in Fig. 1(d) implies the existence of a first peak, which should be located before $5 \mathrm{hr}$. It is clear that glucose cannot produce this first peak, since, at the time of this peak, glucose mass hardly decreases, as can be seen in Fig. 1(a).

To understand the difference between G6P biological data and the calculated curve, we integrated a combined plot in Fig. 2(a) which includes: biological data of cell proliferation (L1) and extracellular glucose concentration (L2), simulation result of cellular glucose uptake speed (L3), experimental G6P concentration (L4) and its predicted curve from 0 to $5 \mathrm{hr}$ (L5), predicted glycogen concentration (L6).

Cellular glucose uptake speed reaches its peak around $7 \mathrm{hr}$, so in this period, cell life activity is most vigorous; correspondingly, cell proliferation curve is in the log phase. After $8 \mathrm{hr}$, cells still uptake much glucose from medium for a long period, but cell proliferation enters the stable phase, where little energy is required; meanwhile, G6P concentration is continually decreasing. This indicates that after $8 \mathrm{hr}$, glucose uptake is not mainly used for cell proliferation, and there should be another destination for these sugar groups. We should not forget that there is another pathway connected with G6P, glycogen metabolism pathway [18,21,22], which is an intracellular sugar storage mechanism. This suggests that, in this phase, glucose is changed to glycogen.

From 5 to $6 \mathrm{hr}$, cell growth needs more sugar than before $5 \mathrm{hr}$, and extracellular glucose supplies almost no glucose; as a consequence G6P concentration decreases. Nevertheless, where does the sugar of G6P come from at $5 \mathrm{hr}$ ? Before $5 \mathrm{hr}$ almost no extracellular glucose is taken in, and no sugar group comes from the gluconeogenesis pathway; indeed concentration of PEP and 3PG, which are essential metabolites on the pathway of gluconeogenesis and glycolysis, are at basal level at $5 \mathrm{hr}$ (Fig. 2(b) and 2(c)), and cells do not maintain stable G6P levels. This suggested that intracellular glycogen provides sugar group to G6P before 5 or $6 \mathrm{hr}$.

\section{Prediction of the glycogen role in cell proliferation:}




\section{(1) In lag phase, glycogen supplies sugar for cell life activity;}

(2) In stable phase, glucose is converted to glycogen, being stored in a cell for its next growth.

In the log phase of the bacterial proliferation, after glycogen decreased, glucose works as the main sugar donor. Based on this prediction, we drew the curve of glycogen concentration tendency (L6 of Fig. 2(a)), which includes three phases roughly. Since G6P is not an intracellular sugar supply, its initial value should be similar to the final experimental data at $11 \mathrm{hr}$ (L3 of Fig. 2(a)). It is clear that glucose cannot produce the first peak of G6P, since, at the time of this peak, extracellular glucose mass hardly decreases at all (L2). Thus, we predict that the first peak, located before $5 \mathrm{hr}$, is caused by glycogen.

\subsection{Experimental confirmation of glycogen concentration, and determination of the location of the predicted peak of G6P}

Subsequently, we ran an experiment to measure cellular glycogen concentration (Fig. 2 (d)), in the same conditions as previous experiments. In this experimental pattern, from 2 to $3 \mathrm{hr}$, glycogen is in the decreasing phase; from 3 to $4 \mathrm{hr}$, the curve is quite flat; and from $4 \mathrm{hr}$, glycogen mass starts to accumulate quickly. Although the time course of the predictive (L5 of Fig. 2 (a)) and biological curve (Fig. 2 (d)) is slightly different, they show a similar tendency. This result is sufficient to prove the correctness of our glycogen prediction.

Between 3 and $4 \mathrm{hr}$, glycogen alternates its function from a sugar donor to a sugar acceptor, that is, sugar group flux direction changes (Fig. 2(d)). Meanwhile, extracellular glucose starts being consumed, though a little; before $3 \mathrm{hr}$ extracellular glucose has not been taken in, and cell proliferation still remains slow. In summary, around $3 \mathrm{hr}$, almost all glycogen has been changed to G6P, and no or very little flows out. Thus, we predict the first peak of intracellular G6P should appear around $3 \mathrm{hr}$ (see G6P predictive curve in Fig. 2(d)).

\section{Discussion}

4.1 Comprehensive understanding on carbohydrate metabolic pathways associated 


\section{with $E$. coli proliferation}

Computational simulation of G6P was integrated with biological data of glucose, glycogen and cell proliferation, into a comprehensive chart in Fig. 3 (left part), and carbohydrate metabolism was divided into four phases; the corresponding sugar group flux balance diagrams are depicted in the right part of Fig. 3.

Phase A: In the first phase, the only sugar donor is intracellular glycogen; extracellular glucose uptake has not begun. Glycogen supplies sugar to G6P; and because cell quick proliferation does not start, less sugar flows to glycolysis and PPP; so G6P reaches its first peak when glycogen curve stops its conversion to G6P.

Phase B: During this short phase, extracellular glucose and intracellular glycogen change their roles: (1) extracellular glucose starts being taken up, as a major sugar group donor; (2) and the function of intracellular glycogen is changed from a sugar group donor to an acceptor.

Phase C: Both extracellular glucose consumption and cell proliferation are in their exponential phase. Glucose is the main energy source for cell division and growth, and simultaneously glycogen serves as a sugar group acceptor as storage. In this phase G6P concentration varies much, as sugar input is compensated by sugar output.

Phase D: From the beginning of this phase, cell proliferation stops, redundant glucose is stored as glycogen. The only sugar flux pathway is from glucose to glycogen in this phase.

Generally the central metabolism pathway of E. coli is associated with cell proliferation. At first, extracellular glucose is not uptake, and glycogen acts as the major sugar donor to supply energy for a cell maintaining its routine life activity. As extracellular glucose starts being imported, glycogen changes its role from a sugar donor to a sugar acceptor as storage, and cell division becomes more active. While cell proliferation enters the log phase, glucose becomes the absolute sugar donor for a cell, and in the meantime more sugar group flows to glycogen for storage. At last, cell growth stops, and the sugar group output destination is glycogen only. Obviously, 
glycogen not only takes part in cell proliferation, but also plays a more important role than previously thought $[20,21,46]$

\subsection{Modeling the role of glycogen}

Based on the analyses above, we updated Eqn (6) by including glycogen metabolic pathway in:

$$
d\left(m_{G 6 P}\right) / d t=v_{g l c}^{\text {input }}+v_{g l g}^{\text {input }}-v^{\text {output }},
$$

where $v_{g l g}^{\text {input }}$ only represents the function of glycogen acting as sugar donor, $v_{g l c}^{\text {input }}$ and $v^{\text {output }}$ are the same as in Eqn (6). When modeling, if we want to have a similar computational curve with the biological plot of glycogen concentration, which includes both decreasing and accumulating parts, a decision mechanism is required. In our culture condition, the limiting nutrient was clearly nitrogen source (Fig. 1(a)) and this limitation lead cells into stationary growing phase. Glucose concentration in the medium keeps decreasing until exhausted after the cells enter the stationary phase. But whether the nitrogen source is the decision mechanism for carbon flux in E. coli has not been confirmed so far, and we are still investigating this topic.

Nevertheless, the partial function of glycogen, supplying sugar, can easily be considered in the context of this paper. Glycogen concentration curve can be calculated by a reverse Gompertz function (Eqn (8)).

$$
m_{g l g}(t)=G\left(1-a_{3} e^{b_{3} e^{c_{3} t}}\right)
$$

where $m_{g l g}(t)$ represents intracellular glycogen concentration, $G=147$ is the initial value of glycogen concentration, and $a_{3}=1, b_{3}=-10, c_{3}=-1.2$ are parameters of Gompertz function. Simulation curve of glycogen as a sugar-donor is shown in Fig. 4(a).

Meanwhile, kinetics of glycogen mass decreasing speed can be achieved by derivative of glycogen mass function. Then the speed of glycogen supply sugar group is given by: 


$$
v_{g \mathrm{lg}}(t)=-k_{3} m_{g \mathrm{gl}}^{\prime}(t)
$$

where $k_{3}=3.5$ has the same function as $k_{1}$, which is a parameter for units normalization.

G6P concentration computational result is available at Fig. 4(b) (for CSML models, see section 2.6). As expected, two peaks appeared, and their locations are also similar with the biological and predictive data (Fig. 2(d)). The computational result of G6P concentration gave another strong support for our prediction that glycogen plays a more important role than previously thought during the process of $E$. coli proliferation.

\section{Conclusions}

This paper illustrates a systems methodology of how the biological fact can be uncovered from experimental data with the support of computational methods. First, through experimental cell proliferation and extracellular glucose concentration data, we calculated the 'second peak' of average cellular G6P concentration, which was caused by glucose and coincident with its experimental result, in spite of a 'hook' difference at about 6 hr. Then, a combinational analysis with biological and computational data was executed to check the 'hook'; we predicted glycogen acts as a major sugar donor and contributes to the 'first peak' in G6P curve, and this prediction was proved by experimental measurement of glycogen concentration.

Reciprocally, the biological glycogen data helped us to determine the more accurate location of the first peak of G6P. As additional evidence, computation also yielded a simulation result of G6P concentration, which has a double peak, by simulating partial function of glycogen as a sugar donor. Finally, based on former results, a new aspect of E. coli central metabolism, with two major sugar donors, extracellular glucose and intracellular glycogen, has emerged. Recently, the property of glycogen has been extensively investigated [20]. E. coli mutants deleted in either pts or pgm genes display glycogenless phenotypes, and these genes showed nonessential for cell growth [18,22]. Glycogen synthesis, however, was reported in relationship with biofilm formation and virulence in Salmonella enteritidis [47] and environmental persistence of Vivrio cholera [48]. It seems that there has been so much left for the new property to be investigated, and the model of this paper is a milestone on the way of our continuing research on the 
role of glycogen in metabolic pathways.

Our achievements: (1) we successfully predicted and proved that glycogen plays a more important role as a sugar group donor in cell proliferation than previously thought. (2) The occurrence and timing of the first peak of G6P concentration curve was also confirmed and slightly adjusted by logical deduction on experimental and simulation results. (3) The HFPN model we set up, not only could help readers to understand the importance of glycogen in cell proliferation, but also displayed a real process of how a biological prediction is stimulated by computational methods. (4) The computational model that_we set up in this study, will be the first part of our future model of the whole metabolism pathway in E. coli. 


\section{Acknowledgements}

This work was supported by a Grant-in-Aid for Scientific Research (A) (22241050) from the Ministry of Education, Culture, Sports, Science and Technology of Japan.

\section{References}

[1] M. Heinemann, U. Sauer, Systems biology of microbial metabolism, Curr. Opin. Microbiol., 13 (2010) 337-343.

[2] C. Chassagnole, N. Noisommit Rizzi, J.W. Schmid, K. Mauch, M. Reuss, Dynamic modeling of the central carbon metabolism of Escherichia coli, Biotechnol. Bioeng., 79 (2002) 53-73.

[3] F.J. Bruggeman, F.C. Boogerd, H.V. Westerhoff, The multifarious short term regulation of ammonium assimilation of Escherichia coli: dissection using an in silico replica, Febs J., 272 (2005) 1965-1985.

[4] J. Yuan, C.D. Doucette, W.U. Fowler, X.-J. Feng, M. Piazza, H.A. Rabitz, N.S. Wingreen, J.D. Rabinowitz, Metabolomics-driven quantitative analysis of ammonia assimilation in E coli, Mol. Syst. Biol., 5 (2009).

[5] O. Kotte, J.B. Zaugg, M. Heinemann, Bacterial adaptation through distributed sensing of metabolic fluxes, Mol. Syst. Biol., 6 (2010).

[6] A. Kremling, K. Bettenbrock, E. Gilles, Analysis of global control of Escherichia coli carbohydrate uptake, BMC Syst. Biol., 1 (2007) 42.

[7] A. Kremling, K. Bettenbrock, E.D. Gilles, A feed-forward loop guarantees robust behavior in Escherichia coli carbohydrate uptake, Bioinformatics, 24 (2008) 704 -710 .

[8] G. Gosset, Improvement of Escherichia coli production strains by modification of the phosphoenolpyruvate: sugar phosphotransferase system, Microb. Cell Fact., 4 (2005) 14.

[9] D.L. Nelson, M.M. Cox, Lehninger Principles of Biochemistry, Fifth Edition, W.H. Freeman, 2007.

[10] M. Arad, B.J. Maron, J.M. Gorham, W.H. Johnson, J.P. Saul, A.R. Perez-Atayde, 
P. Spirito, G.B. Wright, R.J. Kanter, C.E. Seidman, J.G. Seidman, Glycogen Storage Diseases Presenting as Hypertrophic Cardiomyopathy, N. Engl. J. Med., 352 (2005) 362-372.

[11] M.J. Danon, S.J. Oh, S. DiMauro, J.R. Manaligod, A. Eastwood, S. Naidu, L.H. Schliselfeld, Lysosomal Glycogen Storage Disease with Normal Acid Maltase, Neurology, 31 (1981) 51-51.

[12] P.S. Harper, Landmarks in Medical Genetics: Classic Papers With Commentaries, Oxford University Press, 2004.

[13] J. Hay, M.J. Levin, J.M. Sondheimer, R.R. Deterding, Glycogen Storage Diseases, McGraw-Hill, 2007.

[14] J.E. Riggs, S.S. Schochet, L. Gutmann, S. Shanske, W.A. Neal, S. DiMauro, Lysosomal Glycogen Storage Disease Without Acid Maltase Deficiency, Neurology, 33 (1983) 873-873.

[15] T.E. Starzl, A.J. Demetris, M. Trucco, C. Ricordi, S. Ildstad, P.I. Terasaki, N. Murase, R.S. Kendall, M. Kocova, W.A. Rudert, A. Zeevi, D. Van Thiel, Chimerism after Liver Transplantation for Type IV Glycogen Storage Disease and Type 1 Gaucher's Disease, N. Engl. J. Med., 328 (1993) 745-749.

[16] F. Van Hoof, L. Hue, T. de Barsy, P. Jacquemin, P. Devos, H.G. Hers, Glycogen storage diseases, Biochimie, 54 (1972) 745-751.

[17] M. Montero, G. Almagro, G. Eydallin, A.M. Viale, F.J. Muñoz, A. Bahaji, J. Li, M. Rahimpour, E. Baroja Fernández, J. Pozueta Romero, Escherichia coli glycogen genes are organized in a single glgBXCAP transcriptional unit possessing an alternative suboperonic promoter within $\mathrm{glgC}$ that directs glgAP expression, Biochem. J., 433 (2011) 107-117.

[18] M. Montero, G. Eydallin, A.M. Viale, G. Almagro, F.J. Muñoz, M. Rahimpour, M.T. Sesma, E. Baroja Fernández, J. Pozueta Romero, Escherichia coli glycogen metabolism is controlled by the PhoP-PhoQ regulatory system at submillimolar environmental $\mathrm{Mg} 2+$ concentrations, and is highly interconnected with a wide variety of cellular processes, Biochem. J., 424 (2009) 129-141.

[19] C.R. Meyer, M. Borra, R. Igarashi, Y.-S. Lin, M. Springsteel, Characterization of ADP-Glucose Pyrophosphorylase from Rhodobacter sphaeroides 241: Evidence 
for the Involvement of Arginine in Allosteric Regulation, Arch. Biochem. Biophys., 372 (1999) 179-188.

[20] W.A. Wilson, P.J. Roach, M. Montero, E. Baroja Fernández, F.J. Muñoz, G. Eydallin, A.M. Viale, J. Pozueta Romero, Regulation of glycogen metabolism in yeast and bacteria, FEMS Microbiol. Rev., 34 (2010) 952-985.

[21] G. Eydallin, A.M. Viale, M.T. Morán-Zorzano, F.J. Muñoz, M. Montero, E. Baroja-Fernández, J. Pozueta-Romero, Genome-wide screening of genes affecting glycogen metabolism in Escherichia coli K-12, FEBS Lett., 581 (2007) 2947-2953.

[22] G. Eydallin, M. Montero, G. Almagro, M.T. Sesma, A.M. Viale, F.J. Muñoz, M. Rahimpour, E. Baroja-Fernández, J. Pozueta-Romero, Genome-Wide Screening of Genes Whose Enhanced Expression Affects Glycogen Accumulation in Escherichia coli, DNA Res., 17 (2010) 61 -71.

[23] T. Romeo, J. Preiss, Genetic regulation of glycogen biosynthesis in Escherichia coli: in vitro effects of cyclic AMP and guanosine 5'-diphosphate 3'-diphosphate and analysis of in vivo transcripts, J. Bacteriol., 171 (1989) $2773-2782$.

[24] T. Romeo, J. Black, J. Preiss, Genetic regulation of glycogen biosynthesis inEscherichia coli: In vivo effects of the catabolite repression and stringent response systems inglg gene expression, Curr. Microbiol., 21 (1990) 131-137.

[25] T. Romeo, A. Kumar, J. Preiss, Analysis of the Escherichia coli glycogen gene cluster suggests that catabolic enzymes are encoded among the biosynthetic genes, Gene, 70 (1988) 363-376.

[26] G. Stephanopoulos, A.A. Aristidou, J. Nielsen, Metabolic Engineering: Principles and Methodologies, Academic Press, 1998.

[27] H. Harry, Flux analysis and control of the central metabolic pathways in Escherichia coli, FEMS Microbiol. Rev., 19 (1996) 85-116.

[28] Q. Hua, C. Yang, T. Baba, H. Mori, K. Shimizu, Responses of the Central Metabolism in Escherichia Coli to Phosphoglucose Isomerase and Glucose-6-Phosphate Dehydrogenase Knockouts, J. Bacteriol., 185 (2003) $7053-7067$.

[29] D.J. Korz, U. Rinas, K. Hellmuth, E.A. Sanders, W.-D. Deckwer, Simple 
fed-batch technique for high cell density cultivation of Escherichia coli, J. Biotechnol., 39 (1995) 59-65.

[30] D.M. LeMaster, D.M. Kushlan, Dynamical Mapping of E coli Thioredoxin via 13C NMR Relaxation Analysis, J. Am. Chem. Soc., 118 (2011) 9255-9264.

[31] Y. Toya, N. Ishii, K. Nakahigashi, T. Hirasawa, T. Soga, M. Tomita, K. Shimizu, 13C metabolic flux analysis for batch culture of Escherichia coli and its pyk and pgi gene knockout mutants based on mass isotopomer distribution of intracellular metabolites, Biotechnol. Prog., 26 (2010) 975-992.

[32] K. Tuty, M. Ahmad, M.F. Johnjoe, S. Kazuyuki, Modeling and simulation of the main metabolism in Escherichia coli and its several single-gene knockout mutants with experimental verification, Microb. Cell Fact., 9 (2010) 88.

[33] W. Wiechert, M. Möllney, S. Petersen, A.A. de Graaf, A Universal Framework for 13C Metabolic Flux Analysis, Metab. Eng., 3 (2001) 265-283.

[34] W. Wolfgang, 13C Metabolic Flux Analysis, Metab. Eng., 3 (2001) 195-206.

[35] B. Xu, M. Jahic, S. Enfors, Modeling of Overflow Metabolism in Batch and Fed Batch Cultures of Escherichiacoli, Biotechnol. Prog., 15 (1999) 81-90.

[36] M.F. Traxler, D.-E. Chang, T. Conway, Guanosine 3',5'-Bispyrophosphate Coordinates Global Gene Expression During Glucose-Lactose Diauxie in Escherichia Coli, PNAS, 103 (2006) 2374-2379.

[37] M.F. Traxler, S.M. Summers, H. Nguyen, V.M. Zacharia, G.A. Hightower, J.T. Smith, T. Conway, The global, ppGpp mediated stringent response to amino acid starvation in Escherichia coli, Mol. Microbiol., 68 (2008) 1128-1148.

[38] M.F. Traxler, V.M. Zacharia, S. Marquardt, S.M. Summers, H. Nguyen, S.E. Stark, T. Conway, Discretely calibrated regulatory loops controlled by ppGpp partition gene induction across the "feast to famine" gradient in Escherichia coli, Mol. Microbiol., 79 (2011) 830-845.

[39] J. Preiss, E. Greenberg, A. Sabraw, Biosynthesis of Bacterial Glycogen Kinetic Studies of a Glucose-1-Phosphate Adenylyltransferase (EC 27727) from a Glycogen-Deficient Mutant of Escherichia Coli B, J. Biol. Chem., 250 (1975) 7631-7638.

[40] N. Sigal, J. Cattaneo, I.H. Segel, Glycogen accumulation by wild-type and 
uridine diphosphate glucose pyrophosphorylase-negative strains of Escherichia coli, Arch. Biochem. Biophys., 108 (1964) 440-451.

[41] B.L. Wanner, Gene expression in bacteria using TnphoA and TnphoA'elements to make and switch phoA gene, lacZ (op), and lacZ (pr) fusions, Mol. Microbiol. Tech., 3 (1994) 291.

[42] T. Soga, Y. Ohashi, Y. Ueno, H. Naraoka, M. Tomita, T. Nishioka, Quantitative Metabolome Analysis Using Capillary Electrophoresis Mass Spectrometry, J. Proteome Res., 2 (2003) 488-494.

[43] A.K. Laird, Dynamics of Tumour Growth, Br. J. Cancer, 18 (1964) 490-502.

[44] A. D'Onofrio, A general framework for modeling tumor-immune system competition and immunotherapy: Mathematical analysis and biomedical inferences, Phys. D., 208 (2005) 220-235.

[45] H. Matsuno, Y. Tanaka, H. Aoshima, A. Doi, M. Matsui, S. Miyano, others, Biopathways representation and simulation on hybrid functional Petri net, In Silico Biol., 3 (2003) 389-404.

[46] J.V. Lawrence, S. Maier, Correction for the inherent error in optical density readings, Appl. Environ. Microbiol., 33 (1977) $482-484$.

[47] M.A. Bonafonte, C. Solano, B. Sesma, M. Alvarez, L. Montuenga, D. García Ros, C. Gamazo, The relationship between glycogen synthesis, biofilm formation and virulence in Salmonella enteritidis, FEMS Microbiol. Lett., 191 (2000) 31-36.

[48] L. Bourassa, A. Camilli, Glycogen contributes to the environmental persistence and transmission of Vibrio cholerae, Mol. Microbiol., 72 (2009) 124-138. 

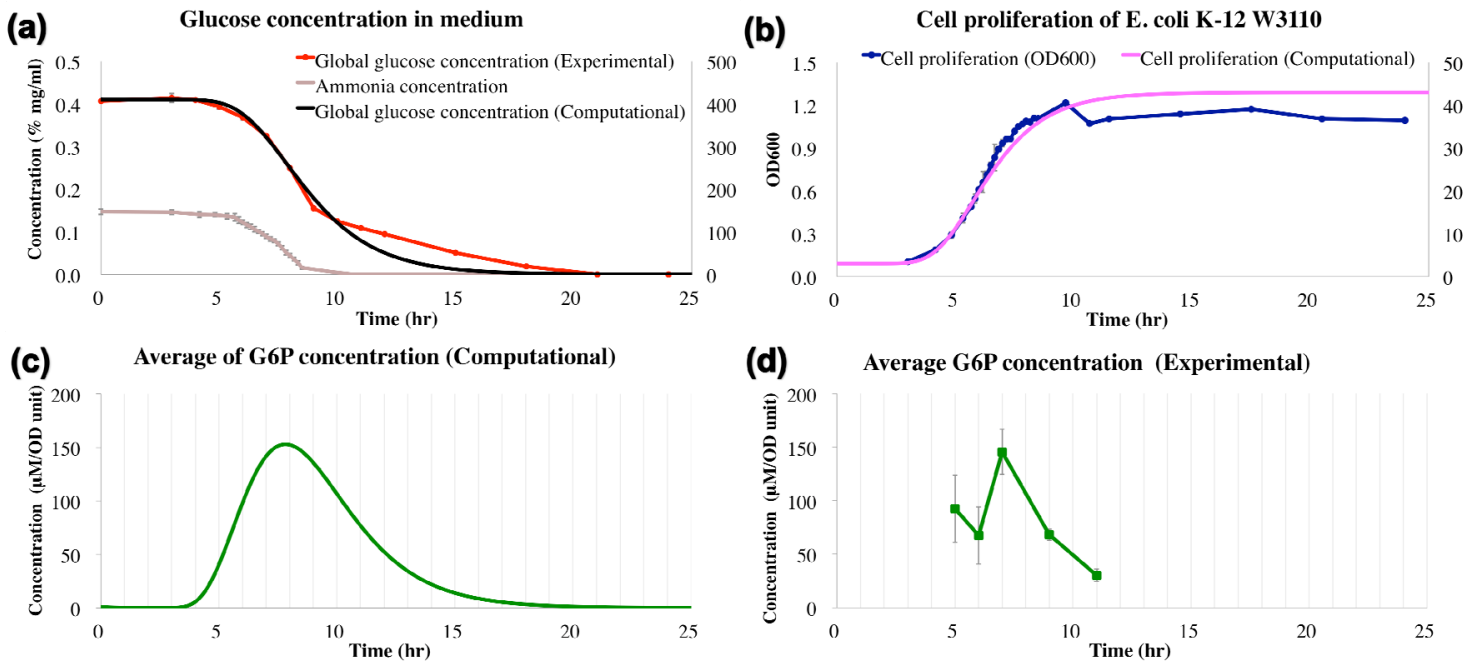

Fig. 1. Experimental and computational data of extracellular glucose concentration, $E$. coli proliferation, and G6P concentration. (a) \& (b) (Biological results): in our culture condition, the limiting nutrient was clearly nitrogen source (ammonia) and this limitation lead cells into stationary growing phase; glucose concentration in the medium kept decreasing until exhausted after the cells entered into the stationary phase. (Computational results): Gompertz function is the major simulation tool. (c) Computational result of G6P average cellular concentration of E. coli, in which extracellular glucose is the sugar donor. (d) Experimental result of G6P average cellular concentration, in which the peak at $7 \mathrm{hr}$ is the so-called 'second peak,' the trough at $6 \mathrm{hr}$ is the so-called 'hook,' in this paper. 
(a)

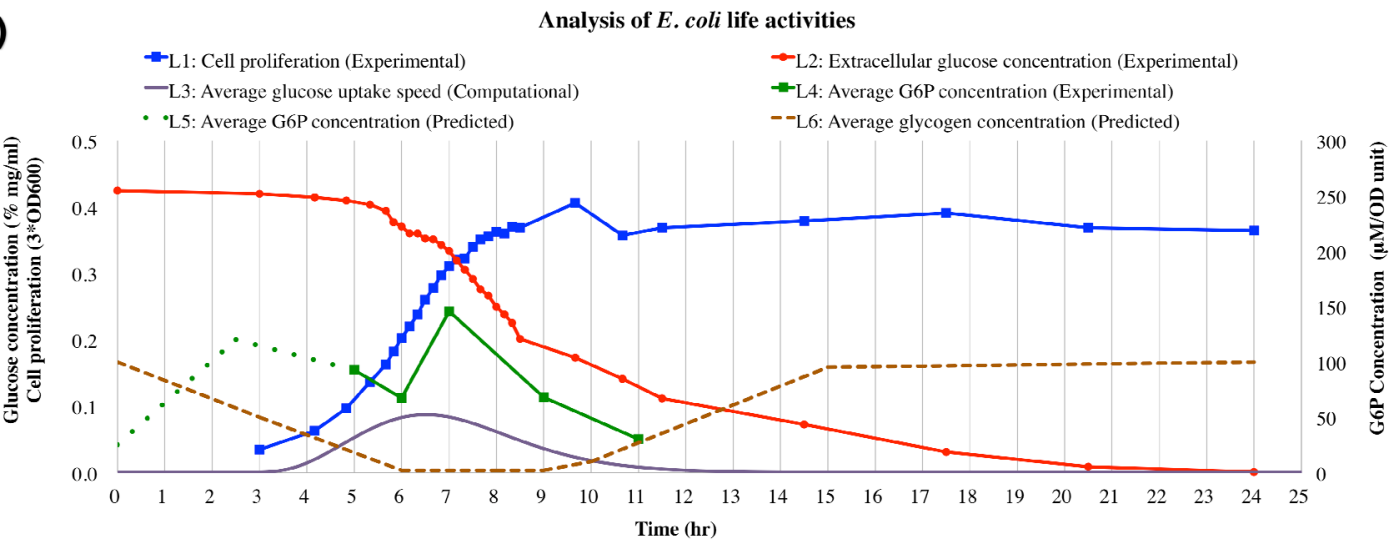

(b)

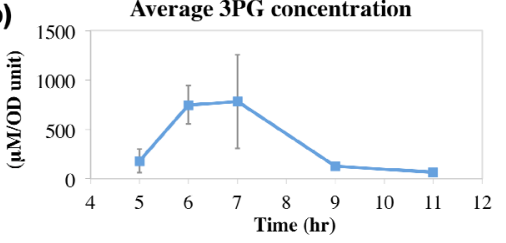

(c)

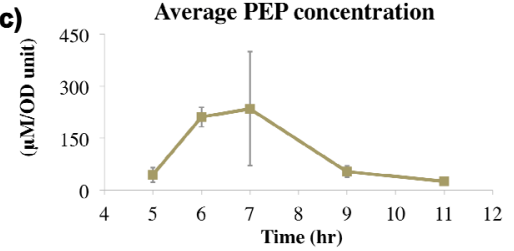

(d)

Average glycogen concentration (Experimental)

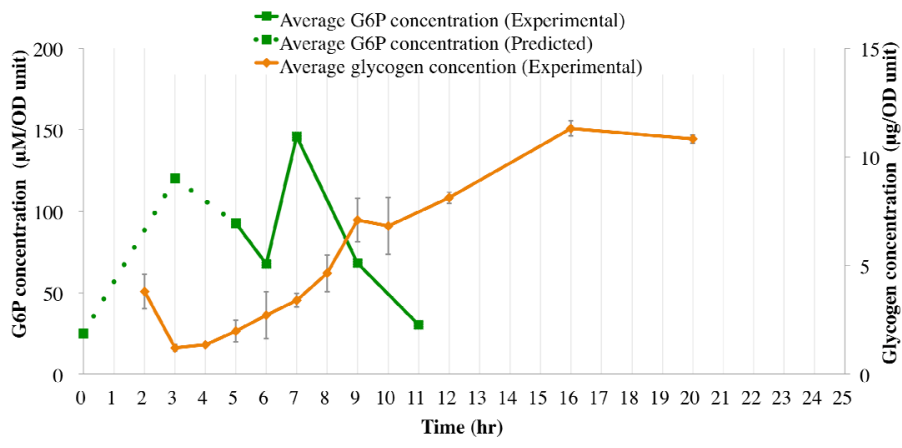

Fig. 2. Glycogen is the main sugar donor during the first phase of bacterial proliferation.

(Experimental results): (L1) is E. coli proliferation; (L2) is glucose concentration in medium; (L4) is G6P average cellular concentration, in which the peak at $7 \mathrm{hr}$ is the so called 'second peak', the trough at $6 \mathrm{hr}$ is the so called 'hook', in this paper. (Computational result): (L3) is extracellular glucose uptake speed of a cell, in which the peak around $7 \mathrm{hr}$ indicates the most vigorous life activity period of E. coli. (Predictions): (L5) is the predicted G6P average cellular concentration, the peak in L1 was the so called the 'first peak' here; (L6) is the predicted glycogen concentration of E. coli, it roughly includes three periods: decrease, bottom phase, and increase. (b) Average concentration of 3PG. (c) Average concentration of PEP. (d) Blank square line represents experimental measure of glycogen cellular concentration. Solid square line is G6P concentration curve, which includes experimentally obtained part (solid line) and predicted part (dotted line), and the position of the 'first peak' has been adjusted according to the glycogen experimental data. 


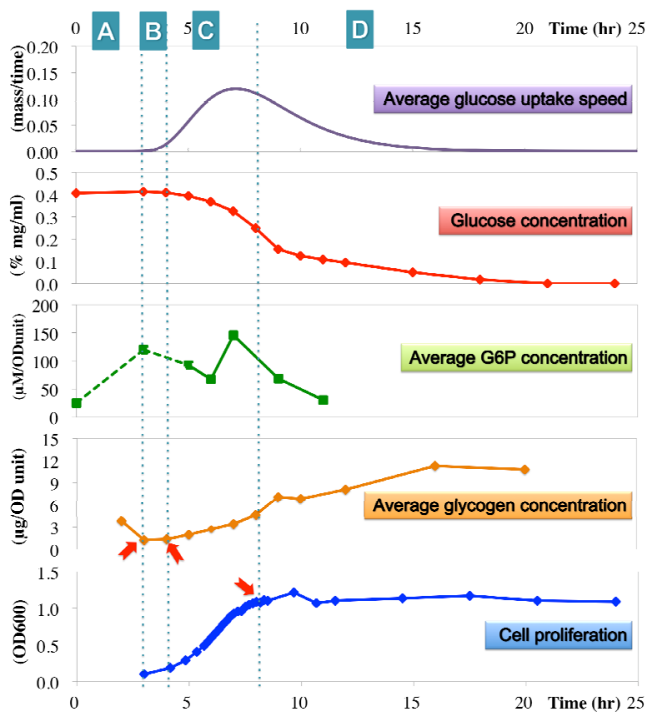

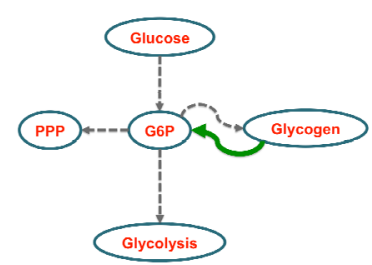

A

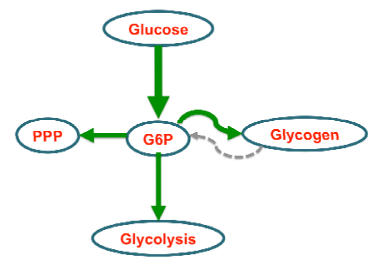

C

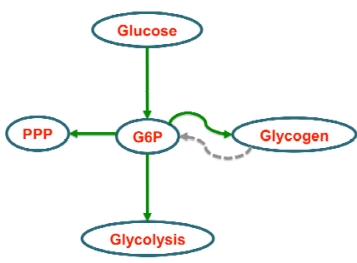

B

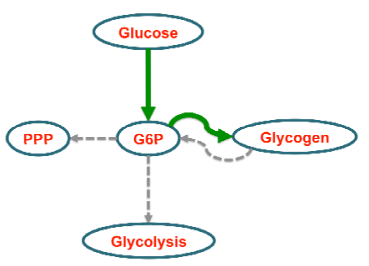

D

Fig. 3. Comprehensive understanding of sugar groups flux around G6P in the process of $E$. coli proliferation. Phase A: In the first phase, the only sugar donor is intracellular glycogen; extracellular glucose uptake has not begun. Glycogen supplies sugar to G6P; and because cell quick proliferation does not start, less sugar flows to glycolysis and PPP; so G6P reaches its first peak when glycogen curve stops its conversion to G6P. Phase B: During this short phase, extracellular glucose and intracellular glycogen change their roles: (1) extracellular glucose starts being taken up, as a major sugar-group donor; (2) and the function of intracellular glycogen is changed from a sugar-group donor to an acceptor. Phase C: Both extracellular glucose consumption and cell proliferation are in their exponential phase. Glucose is the main energy source for cell division and growth, and simultaneously glycogen serves as a sugar group acceptor as storage. In this phase G6P concentration varies much, as sugar input is compensated by sugar output. Phase D: From the beginning of this phase, cell proliferation stops, redundant glucose is stored as glycogen. The only sugar flux pathway is from glucose to glycogen in this phase. 
(a)

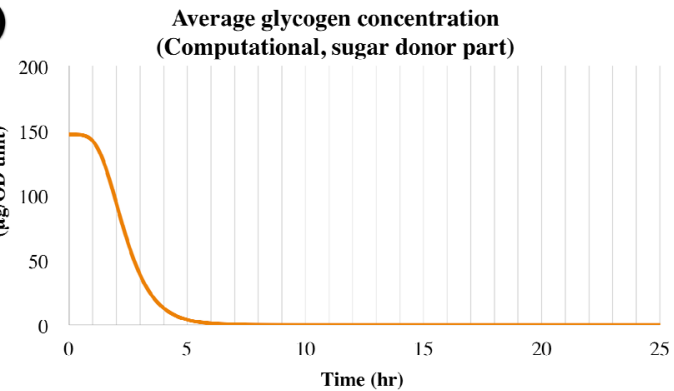

(b) Average G6P concentration (Computational, with double sugar donors)

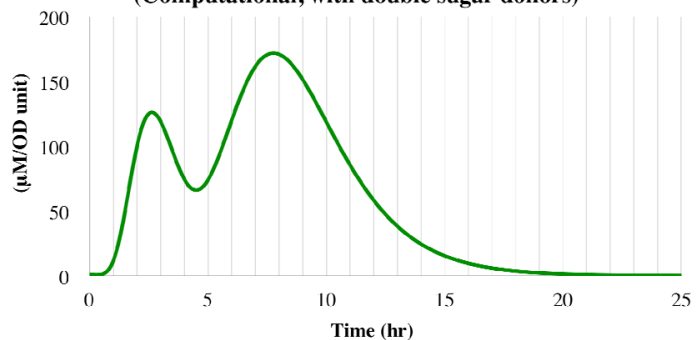

Fig. 4: Computational results of glycogen concentration (sugar donor part) and subsequent

G6P concentration. (a) Computational results of glycogen concentration (sugar donor part). (b) Computational G6P concentration curve displays two peaks, due to intracellular glycogen (the first peak) and extracellular glucose (the second peak) respectively. 